\title{
Computer-Aided Design in Electromagnetics - the Case for Surface Impedance Boundary Conditions
}

\author{
Nathan IDA ${ }^{1}$, Luca Di RIENZO ${ }^{2}$, Sergey YUFEREV ${ }^{3}$ \\ ${ }^{1}$ The University of Akron, Department of Electrical and Computer Engineering, Akron, Ohio \\ 44325-3904 USA \\ ${ }^{2}$ Politecnico di Milano, Department of Electrical Engineering, 32-20133 Milano, \\ ${ }^{3}$ Infineon Technologies, Siemensstrasse 2, Villach 9500, Austria \\ ida@uakron.edu
}

\begin{abstract}
Surface impedance boundary conditions (SIBCs) have been successfully used for over 70 years in both analytical and numerical computation. With the need to model increasingly complex geometries and smaller artifacts, its importance in computer-aided design of electromagnetic devices has become prominent. High frequency SIBCs have been particularly successful because of the minimal penetration of electromagnetic fields in conductors and lossy dielectrics. SIBCs based on the skin depth have also been used although these have been limited to the first order (Leontovich) condition and Leontovich-like conditions. Little has been done in incorporating second order SIBCs and higher. A general method of derivation of SIBCs of arbitrary order is presented here and shown to apply to low frequency power structures including electric machines, transmission lines and nondestructive testing of materials. The proposed SIBCs are universally applicable and the order of the SIBC allows control of errors in design. Whereas low order SIBCs apply to classical flat surfaces and perpendicular diffusion, higher order conditions take into account curvatures and lateral diffusion of fields as well. Results shown include transmission line parameters, eddy current testing and other power applications in which they contribute to speed and accuracy of the design. In some cases, the use of SIBCs is not only possible but rather is critical to the very ability to obtain an acceptable design.
\end{abstract}

Index Terms-Boundary element methods, Diffusion processes, Electromagnetics, Numerical analysis, Surface impedance boundary conditions.

\section{INTRODUCTION}

The concept of surface impedance has been coined in 1938 as a means of analyzing problems of reflection from and shielding by conducting surfaces [1]. Shortly after, the first and perhaps the most widely used SIBC, due to Leontovich has been initially developed in an attempt to simplify the analysis of propagation of electromagnetic waves over the surface of the earth [2]. But it was quickly realized that its applicability is universal and since then, surface impedance boundary conditions have seen a steady increase in applications and acceptance and the development of new types and new methods of computation. The application to scattering and propagation of electromagnetic waves has initially been the preferred target for the method, especially in theoretical studies but the advent of numerical computation has changed the focus. It was immediately recognized that SIBCs can contribute considerably to design in conjunction with numerical methods by reducing the computational load by eliminating conducting bodies from the analysis and replacing them with the surface impedance. In certain applications the use of SIBCs is essential without them the model is too large for any meaningful solution. In others it allows the model to concentrate on details without encumbering it with computation in regions of no interest. For these reasons and others, the concept of surface impedance boundary condition has seen a resurgence that coincided with the development of design and analysis methods based on numerical approaches [3]. The method has been applied in every conceivable domain for design of devices ranging from electric machines to antennas and everything in between [3-9]. As numerical models are relied upon to solve an ever increasing number of physical problems with increasing size, resolution and accuracy, SIBCs are gaining acceptance as a simple, reliable, analytical method relatively easily integrated within numerical design tools. A clear indication of this trend is the incorporation of SIBCs in commercial design tools in electromagnetics.

In spite of this tendency and the many developments in SIBCs, most of the applications use first order approximations. In addition, the extension of the approximations to low frequency has been done more or less on an ad-hoc basis. That meant that much of the work involved in adapting a numerical program to integrate SIBCs was application specific, impeding its use on a wider scale. However, SIBCs can be based on known quantities such as the skin depth and characteristic physical dimensions of the problem such as curvature and thickness, allowing one not only to design universally applicable and adaptive SIBCs but, more importantly, to predict their performance based on truncation errors that can themselves be associated with the basic parameters of the problem $[3,10]$.

\section{THE CONCEPT}

To fully understand the scope of surface impedance boundary conditions on can start with perfect conductors. In a perfect electric conductor field penetration is zero at all frequencies and hence the perfect conductor is excluded from computation entirely. But its effects must be taken into account by imposition of boundary conditions in lieu of the perfect conductor. These boundary conditions take into 
account reflections so that the field in the space near the conductor is physically correct. Since in nature there are no true perfect conductors, one must assume penetration of fields in conducting media, a process defined by the skin effect in the medium and by Snell's law of refraction. Skin depth defines the diffusion process whereas Snell's law indicates that for highly conducting bodies diffusion into the conductor occurs perpendicular to the surface, almost entirely independent of the incidence angle. If the skin depth is small, or alternatively, if the conductor is thick in comparison to the skin depth, the field in the conductor will be completely attenuated within the conductor. This means the field in the conducting medium is a highly damped plane wave and hence its distribution in the conductor is known a priori. Because the electromagnetic field is continuous at the interface of the conductor, the wave impedance in the conductor may be taken as the ratio of the tangential electric and tangential magnetic fields at the interface. As a reference, at a conducting interface coincident with the $x-y$ plane the wave impedance is:

$$
\frac{E x}{H y}=\sqrt{\frac{j \omega \mu_{c}}{\sigma_{c}+j \omega \varepsilon_{c}}} \approx \frac{1+j}{2} \omega \mu \delta \quad[\Omega]
$$

This relation is only valid at the interface and assumes a high-loss medium for the conductor, that is, that at the frequency of interest, $\sigma_{c} \gg \omega \varepsilon_{c}$ with the index $c$ indicating the conductor. The relation in Eq. (1) is assumed constant on the surface of the conductor and may be taken as a boundary condition in the governing equation and by so doing, removing the conductor from consideration. The condition in (1) is often called the Leontovich condition [2]. It has been derived in the frequency domain, but time domain equivalent formulas also exist [3,5,11-14].

The approximation in (1) is explicit in terms of skin depth and the attenuation in the conducting medium is depth dependent. One can then argue that the expected error in its use should be proportional to the ratio $\delta / D$ where $\delta$ is the skin depth and $D$ is the characteristic size of the conductor. That is, the first order approximation should have error of the order of $(\delta / D)^{2}$. The more important question is how can one reduce this error to ensure more accurate solution and design. A related issue has to do with the fact that (1) is derived from a plane wave impinging on a flat surface and hence the approximation is only valid for flat (or locally flat) conducting surfaces.

The obvious answer is to increase the order of approximation. This has been originally attempted through use of the perturbation method of Rytov [15]. In this approach, the electric and magnetic fields were assumed to vary exponentially inside the conductor and were written as power series expansions in terms of the skin depth. The coefficients of the expansion were evaluated by equating terms of equal powers of the skin depth in the internal and external approximations. This approach leads to arbitrary order SIBCs with the error decreasing with the increase in order. The zeroth order Rytov approximation corresponds to the perfect electric conductor (PEC) whereas the first order corresponds to the Leontovich approximation in (1). Although one can write arbitrary order approximations, the complexity increases quickly and the reduction in error becomes insignificant past the third order. In addition, the method takes into account the curvature of the surface $\left(2^{\text {nd }}\right.$ order Rytov approximation and above) and field variations along the surface $\left(3^{\text {rd }}\right.$ order approximations and above). As an important added advantage, the method also allows calculation of fields and related quantities in the conductor itself based on the analytical distribution within it.

Based on Rytov's approach and using a local orthogonal coordinate system on the surface of the conductor where we denote the tangential coordinates as $\xi_{1}$ and $\xi_{2}$ (the third coordinate $\eta$ points into the conductor) and their corresponding radii of curvature as $d_{1}$ and $d_{2}$, the general expression of the SIBC for up to $3^{\text {rd }}$ order approximation has been derived as follows $[3,15]$ :

$$
\begin{gathered}
E_{\xi_{k}}=(-1)^{3-k} \frac{1+j}{2 \theta} \mu \omega \delta\left(\left(1+\frac{1-j}{4 \theta} \delta\left(d_{3-k}^{-1}-d_{k}^{-1}\right)\right) H_{\xi_{3-k}}+\right. \\
\frac{\delta^{2}}{2 j \theta} \frac{3 d_{k}^{2}-d_{3-k}^{2}-2 d_{k} d_{3-k}}{8 d_{k}^{2} d_{3-k}^{2}} H_{\xi_{3-k}}+ \\
\left.\frac{\delta^{2}}{4 j \theta}\left(-\frac{\partial^{2} H_{\xi_{3-k}}}{\partial \xi_{k}^{2}}+\frac{\partial^{2} H_{\xi_{3-k}}}{\partial \xi_{3-k}^{2}}+2 \frac{\partial^{2} H_{\xi_{3}}}{\partial \xi_{3-k} \partial \xi_{k}^{2}}\right)\right)
\end{gathered}
$$

where:

$$
\theta=\sqrt{1+\frac{j \varepsilon \omega}{\sigma}} ; \delta=\sqrt{\frac{2}{\omega \sigma \mu}} .
$$

The SIBC can be derived in the time domain directly or can be obtained from (1) using inverse the Laplace transform and written in the form of the convolution with respect to time [3]:

$$
\left.E_{x}\right|_{\text {interface }}=-\left.\sqrt{\mu /\left(4 \pi \sigma t^{3}\right)} * H_{y}\right|_{\text {interface }} .
$$

Without showing the details [3], the time-domain equivalent of (2) is:

$$
\begin{gathered}
E_{\xi_{k}}=(-1)^{3-k}\left(T_{1} * H_{\xi_{3-k}}+\left(d_{3-k}^{-1}-d_{k}^{-1}\right) T_{2} * H_{\xi_{3-k}}+\right. \\
\frac{3 d_{k}^{2}-d_{3-k}^{2}-2 d_{k} d_{3-k}}{8 d_{k}^{2} d_{3-k}^{2}} T_{3}^{*} H_{\xi_{3-k}}+ \\
\left.\frac{1}{2} T_{3} *\left(-\frac{\partial^{2} H_{\xi_{3-k}}}{\partial \xi_{k}^{2}}+\frac{\partial^{2} H_{\xi_{3-k}}}{\partial \xi_{3-k}^{2}}+2 \frac{\partial^{2} H_{v_{3}}}{\partial \xi_{3-k} \partial \xi_{k}^{2}}\right)\right)
\end{gathered}
$$

Here * denotes a time domain convolution product and the time-dependent functions $T_{k}$ are defined as follows:

$$
\begin{gathered}
T_{1}(t)=\left(\frac{\mu}{\varepsilon}\right)^{1 / 2}\left(\delta(t)+\frac{\sigma}{2 \varepsilon}\left(I_{1}\left(\frac{\sigma t}{2 \varepsilon}\right)-I_{0}\left(\frac{\sigma t}{2 \varepsilon}\right)\right) \exp \left(-\frac{\sigma t}{2 \varepsilon}\right)\right), \\
\left.T_{2}(t)=\varepsilon^{-1} \exp \left(-\frac{\sigma t}{\varepsilon}\right)\right), \\
T_{3}(t)=t(\varepsilon \sqrt{\varepsilon \mu})^{-1}\left(I_{0}\left(\frac{\sigma t}{2 \varepsilon}\right)-I_{1}\left(\frac{\sigma t}{2 \varepsilon}\right)\right) \exp \left(-\frac{\sigma t}{2 \varepsilon}\right),
\end{gathered}
$$

where $I_{n}(x)$ is the modified Bessel function of order $n$ 
and $\delta(t)$ is the Dirac delta function.

Because of the way the SIBCs are derived, one can select the order of approximation by simply retaining the corresponding terms in the general relation in (2) or (5).

For SIBCs, either in the frequency or time domains to be useful, they must be properly formulated within the numerical method in which they are used since by themselves they are merely relations between the electric and magnetic fields. Formulations are specific to the method used but a variety of formulations have been developed for use with boundary integral methods, finite difference methods, finite integration techniques, finite difference time domain methods and finite elements [3,4,8-9,11,12,16-21]. In a broad view of the application of SIBCs, all that is required is the formulation of (2) or (5) within a numerical method of choice.

\section{AppliCATION To COMPUTATION AND DESIGN}

\section{A. P.u.l. parameters in multi-conductor transmission lines}

High order SIBCs, coupled to the Boundary Element Method (BEM) applied to the computation of the impedance matrix of multi-conductor transmission lines (MTLs) is presented next $[3,16]$. The formulation applies to the calculation of per-unit-length (p.u.1.) parameters of lossy MTLs of arbitrary cross sections.

The geometry consists of $\mathrm{N}$ infinitely long parallel conductors in any configuration. The conductors are made each of a linear nonmagnetic material of permeability $\mu_{i}$, conductivity $\sigma_{i}$ and permittivity $\varepsilon_{i}$. The fields are assumed time-harmonic and represented as phasors. The conductors are described in a Cartesian system with the conductors parallel to the $\mathrm{x}$-axis and, necessarily, we assume conductivities to be high so that the skin effect approximation applies. Each conductor carries a current $I_{i}$. The formulation is based on the implementation of (2) together with the use of the magnetic vector potential in a boundary element approach [16]. The formulation is based on Poisson's equation in the conducting region and in space, with the magnetic vector potential written as a sum of a source and an eddy-current component. With the electric field intensity:

$$
\mathbf{E}=-j \omega \mathbf{A}-\nabla V
$$

The source and eddy-current components are [22]

$$
\nabla V=-j \omega \mathbf{A}^{s} \quad \mathbf{E}=-j \omega \mathbf{A}^{e}
$$

where $\mathbf{A}=\mathbf{A}^{s}+\mathbf{A}^{e}$. Through direct use of Maxwell's equations the equations inside in the conductor can be written as:

$$
\nabla^{2} \mathbf{A}^{e}=j \omega \sigma \mathbf{A}^{e}
$$

whereas outside the conductor $\nabla^{2} \mathbf{A}=0$. Applying the boundary integral method [23] results in the following integral equation over the contours of the cross-section of the conductors (in two dimensions):

$$
\begin{aligned}
& \tilde{c}(\xi) A(\xi, \eta, \omega)+\sum_{i=1}^{N} \oint_{L_{i}} A\left(\xi^{\prime}, \eta, \omega\right) \frac{\partial G\left(\xi, \xi^{\prime}\right)}{\partial \vec{n}^{\prime}} d \xi^{\prime} \\
& =\sum_{i=1}^{N} \oint_{L_{i}} G\left(\xi, \xi^{\prime}\right) \frac{\partial A\left(\xi^{\prime}, \eta, \omega\right)}{\partial \vec{n}^{\prime}} d \xi^{\prime}
\end{aligned}
$$

where $\xi$ and $\eta$ are the surface coordinates described above, $\tilde{c}$ is a coefficient (equal to $1 / 2$ for smooth conductors) and $G\left(\xi, \xi^{\prime}\right)$ is the fundamental solution for the 2-D Laplace equation. The magnetic vector potential is the sum of the source and eddy-current components and the symbol " $\sim$ indicates normalized (non-dimensional) quantities [3]. Substituting first $\mathbf{A}=\mathbf{A}^{s}+\mathbf{A}^{e}$ then following the Rytov approach, the terms of expansion at the surface are obtained as follows:

$$
\left.A^{e}(\xi, \omega)\right|_{\eta=0}=F^{(m)}\left[\left.\frac{\partial A^{e}(\xi, \omega)}{\partial \eta}\right|_{\eta=0}\right]
$$

where $m$ is the order of the expansion. For $m=3$ :

$$
\begin{aligned}
& F^{(3)}[g]=-\left\{\left(j \omega \sigma_{i} \mu_{i}\right)^{-1 / 2} g+\frac{1}{2 d}\left(j \omega \sigma_{i} \mu_{i}\right)^{-1} g+\right. \\
& \left.\frac{3}{8 d^{2}}\left(j \omega \sigma_{i} \mu_{i}\right)^{-3 / 2} g+\frac{1}{2}\left(j \omega \sigma_{i} \mu_{i}\right)^{-3 / 2} \frac{\partial^{2} g}{\partial \xi^{2}}\right\}
\end{aligned}
$$

where $d$ is the local radius of curvature and $g$ a generic function. The first term of (14) is F1 and represents the Leontovich condition. The second term is the correction for the $2^{\text {nd }}$ order approximation (so-called Mitzner condition). The errors for the $1^{\text {st }}, 2^{\text {nd }}$ and $3^{\text {rd }}$ order approximations are $O\left(\delta_{i}^{2} / D_{i}^{2}\right), O\left(\delta_{i}^{3} / D_{i}^{3}\right)$ and $O\left(\delta_{i}^{4} / D_{i}^{4}\right)$, respectively. To calculate the line parameters one requires the surface current density on the conductors and its integral around the circumference provides the total current in the conductor as:

$$
\begin{aligned}
& \oint_{L_{i}}\left(\left.\frac{\partial A^{e}(\xi, \eta, \omega)}{\partial \eta}\right|_{\eta=0}\right) d \xi=I_{i} \\
& \left\{\begin{array}{c}
\frac{\partial}{\partial \tilde{\eta}}\left(\left(1+\alpha T^{*} \tilde{T}\right) \frac{\partial \tilde{H}_{\xi_{k}}}{\partial \tilde{\eta}}\right)=\sigma_{\infty} \mu \frac{\partial \tilde{H}_{\xi_{k}}}{\partial \tilde{t}} \\
c \rho \frac{\partial T}{\partial t}=\chi \sigma_{\infty} \frac{\partial}{\partial \eta}\left(\frac{T}{1+\alpha T} \frac{\partial T}{\partial \eta}\right)+\sigma^{-1} \sum_{i=1}^{2}\left(\frac{\partial H_{\xi_{i}}}{\partial \eta}\right)^{2}
\end{array}\right.
\end{aligned}
$$

Once the solution for the magnetic vector potential is known and the currents were computed one can calculate the terms of the mutual resistance and mutual inductance in the impedance matrix:

$$
\frac{d}{d x} \mathbf{V}(x)=-\mathbf{Z} \cdot \mathbf{I}(x)
$$


where $\mathbf{V}$ and $\mathbf{I}$ are the column vectors of voltages (referred to a reference conductor) and currents respectively.

The left hand side becomes:

$$
j \omega A^{s}=-\frac{\partial V}{\partial x} .
$$

The line self-impedance $Z_{i i}$ and consequently p.u.l. resistances $R_{i i}$ and inductances $L_{i i}$ are obtained as

$$
Z_{i i}=R_{i i}+j \omega L_{i i}=\sum_{k=0, i} \frac{j \omega A_{k}^{s}}{I_{i}},
$$

where the sum over $k$ takes into account the signal conductor $i$ and the reference conductor 0 , being $I_{0}=-I_{i}$. Mutual impedance between conductors $i$ and $j$ is calculated as follows:

$$
Z_{i j}=R_{i j}+j \omega L_{i j}=\sum_{k=i, j} \frac{j \omega A_{k}^{s}}{I_{i}} .
$$

Perhaps the most critical issue in the use of SIBCs is the error incurred in their use. To exactly quantify errors one requires analytical solutions. However relative errors are also useful and in the case of SIBCs the relative errors between various orders of approximation and with respect to numerical solutions without SIBCs provide an indication of convergence towards the "exact" solution. It should also be remembered that errors in computation, and therefore in design, come from various sources including geometry and numerical model and that the errors due to the approximate boundary condition is only one source. To get some insight into this issue, consider Fig. 1, which shows the relative error in the calculation of the line parameters for two parallel copper wires, $2 \mathrm{~mm}$ in diameter and $4 \mathrm{~mm}$ apart. Clearly the error in the $1^{\text {st }}$ order approximation is not acceptable whereas the third order provides much better results. To calculate the errors, the same problem is solved using a finite element program with sufficiently fine discretization for the solution to be considered "exact" for comparison purposes.

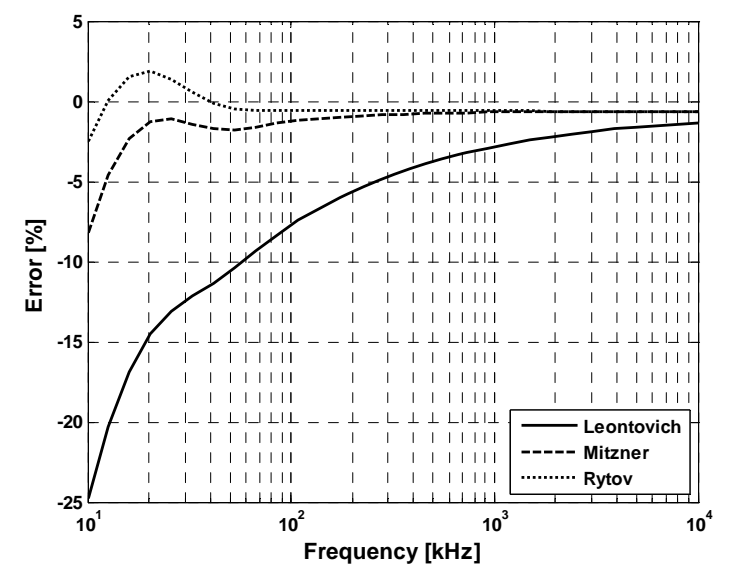

Figure 1. Relative error in computation of the p.u.l. resistance for two identical circular copper conductors of diameter $D=2 \mathrm{~mm}$ and distance between centers equal to $4 \mathrm{~mm}$.

It should also be noted here that SIBCs not only allow reduction of errors but can in fact predict the error a-priori, that is, one can decide before hand the order of the SIBC to produce a given level of error. The prediction is based on the ratio $\delta / D$ discussed above and allows one to decide tailor an appropriate order of approximation for the specific problem at hand rather than using a trial and error [24]. The procedure relies on the fact that the errors for the $1^{\text {st }}, 2^{\text {nd }}$ and $3^{\text {rd }}$ order approximations are $O\left(\delta_{i}^{2} / D_{i}^{2}\right), O\left(\delta_{i}^{3} / D_{i}^{3}\right)$ and $O\left(\delta_{i}^{4} / D_{i}^{4}\right)$, respectively and hence knowledge of the skin depth and the characteristic dimension of the structure can be used to predict the error.

More significant in the design process is the ability to compute the parameters for more complex configurations and over a frequency range. An example is shown in Fig. 2 showing a three-phase power cable with an outer aluminum shield of radius $100 \mathrm{~mm}$ (conductivity $3.6 \times 10^{7} \mathrm{~S} / \mathrm{m}$ ), each copper conductor with radius $17 \mathrm{~mm}$ (conductivity $5.8 \times 10^{7}$ $\mathrm{S} / \mathrm{m}$ ) and distance between centers of $51 \mathrm{~mm}$. The p.u.l. mutual resistance and mutual inductance as functions of frequency are shown in Fig. 3. The p.u.l. self resistance and self inductance of conductor 1 are shown in Table I comparing the calculation using SIBCs in conjunction with the boundary element method with direct calculation using finite elements at a number of frequencies. As expected, larger errors occur at lower frequencies. The erros in calculation of self inductance are lower than those seen in the calculation of self resistance. Calculations for the other conductors show similar results with similar errors.

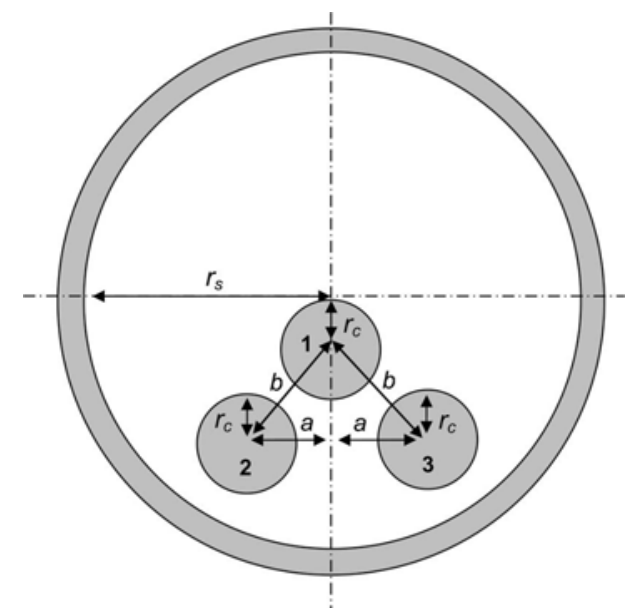

Figure 2. Simulated three-phase power cable $\left(\mathrm{r}_{\mathrm{s}}=100 \mathrm{~mm}, \mathrm{r}_{\mathrm{c}}=17 \mathrm{~mm}\right.$, $\mathrm{a}=1.5 \mathrm{r}_{\mathrm{c}}, \mathrm{b}=3 \mathrm{r}_{\mathrm{c}}$
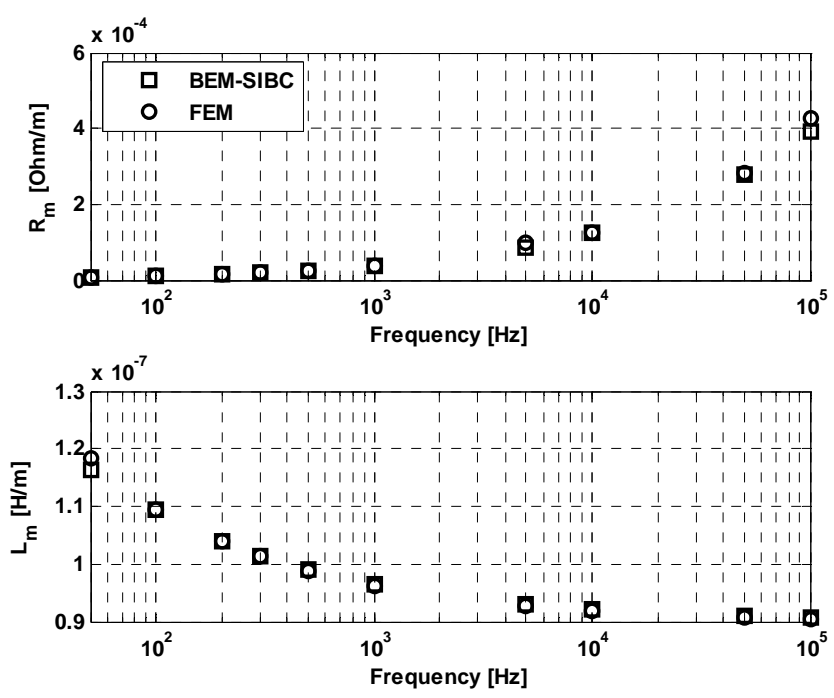

Figure 3. P.u.l. mutual-resistance and mutual-inductance between conductors No. 1 and No. 2 vs. frequency, for the three-phase power cable of Fig. 2. 
TABLE I. P.U.L. SELF RESISTANCE AND SELF INDUCTANCE FOR CONDUCTOR NO 1, COMPARISON WITH FEM CALCULATIONS.

\begin{tabular}{|c|c|c|c|c|}
\hline & \multicolumn{2}{|c|}{ BEM-SIBC } & \multicolumn{2}{c|}{ FEM } \\
\hline $\begin{array}{l}\text { Freq.[ } \\
\text { Hz] }\end{array}$ & $\mathbf{R}_{\mathbf{1 1}}[\Omega / \mathbf{m}]$ & $\mathbf{L}_{\mathbf{1 1}}[\mathrm{H} / \mathbf{m}]$ & $\mathbf{R}_{\mathbf{1 1}}[\boldsymbol{\Omega} / \mathbf{m}]$ & $\mathbf{L}_{\mathbf{1 1}}[\mathrm{H} / \mathbf{m}]$ \\
\hline $\mathbf{5 0}$ & $3.2534 \mathrm{E}-5$ & $3.8605 \mathrm{E}-7$ & $3.3674 \mathrm{E}-5$ & $3.8828 \mathrm{E}-7$ \\
\hline $\mathbf{1 0 0}$ & $4.5510 \mathrm{E}-5$ & $3.6337 \mathrm{E}-7$ & $4.6398 \mathrm{E}-5$ & $3.6302 \mathrm{E}-7$ \\
\hline $\mathbf{2 0 0}$ & $6.4437 \mathrm{E}-5$ & $3.4360 \mathrm{E}-7$ & $6.4894 \mathrm{E}-5$ & $3.4340 \mathrm{E}-7$ \\
\hline $\mathbf{3 0 0}$ & $7.8817 \mathrm{E}-5$ & $3.3449 \mathrm{E}-7$ & $7.9249 \mathrm{E}-5$ & $3.3437 \mathrm{E}-7$ \\
\hline $\mathbf{5 0 0}$ & $1.0174 \mathrm{E}-4$ & $3.2524 \mathrm{E}-7$ & $1.0214 \mathrm{E}-4$ & $3.2515 \mathrm{E}-7$ \\
\hline $\mathbf{1 0}^{\mathbf{3}}$ & $1.4516 \mathrm{E}-4$ & $3.1525 \mathrm{E}-7$ & $1.4440 \mathrm{E}-4$ & $3.1574 \mathrm{E}-7$ \\
\hline $\mathbf{1 0}^{\mathbf{4}}$ & $4.5792 \mathrm{E}-4$ & $3.0011 \mathrm{E}-7$ & $4.5762 \mathrm{E}-4$ & $3.0004 \mathrm{E}-7$ \\
\hline $\mathbf{1 0}^{\mathbf{5}}$ & $1.4812 \mathrm{E}-3$ & $2.9511 \mathrm{E}-7$ & $1.4492 \mathrm{E}-3$ & $2.9506 \mathrm{E}-7$ \\
\hline
\end{tabular}

\section{B. Transient scattering from a cylindrical conductor}

As an example of the use of the surface impedance boundary condition in the time domain, consider the transient scattering from the circular cylinder shown in Fig. 4. The electromagnetic wave propagates from the left towards the lossy cylinder and the magnetic field (or current) is calculated at the surface of the conductor using the finite difference time-domain (FDTD) method. The process here is to start with the FDTD approximation in air:

$$
\begin{gathered}
\partial H_{y} / \partial t=-\mu_{0}^{-1}\left(\partial E_{x} / \partial z-\partial E_{z} / \partial x\right) \\
\partial E_{x} / \partial t=-\varepsilon_{0}^{-1} \partial H_{y} / \partial z ; \partial E_{z} / \partial t=\varepsilon_{0}^{-1} \partial H_{y} / \partial x
\end{gathered}
$$

The FDTD approximation in the conductor using the FDTD stencil at the surface of the conductor is replaced with the approximation in (5). The SIBC is discretized using the FDTD process in curved boundaries resulting in a set of update FDTD equations in air, that exclude the conductor itself. Details of the formulation can be found in [3,25]. Fig. 5 shows the total magnetic field at one point on the cylinder (point A) in terms of normalized time for a TE wave and compares the results for first order (Leontovich) SIBC and $3^{\text {rd }}$ order Rytov SIBC together with the perfect conductor solution. Conductivity of the cylinder is $0.01 \mathrm{~S} / \mathrm{m}$ and the ratio of skin depth and diameter of the cylinder $p=\delta / D=$ 0.5 . In this case, the condition that skin depth be very small compared with the characteristic size of the conductor is not satisfied so we expect relatively large errors when using low order SIBCs. This can be seen in Fig. 5. The perfect conductor approximation is not acceptable for solution because of the errors involved. The Leontovich order approximation is better but exhibits considerable errors. A much better solution is obtained with the $3^{\text {rd }}$ order Rytov approximation. It should be noted however that all three methods are identical in terms of discretization since they all exclude the conductor from the computational space.

The FDTD method is most often used in high frequency applications but no such assumption is required here. The SIBC performs based on the relative ratio $\delta / D$ and the frequency defines this ratio. A highly conductive medium will allow use of the SIBC at lower frequencies whereas at higher frequencies the method can be applied equally well to lossy dielectrics. This particular example shows the results in two dimensions by assuming the conductor to be infinite. Three dimensional solutions are equally simple with allowance for the necessary change in the FDTD approximation. However, care must be taken when sharp corners and edges are involved since these create singularities and must be treated with SIBCs appropriate for them $[13,14,26]$. In some cases, because the singularities are local it is sufficient to "round" the corners and still obtain a proper solution. In still other cases, reasonable results can be obtained by simply ignoring the singularities and as a consequence incurring an additional error.

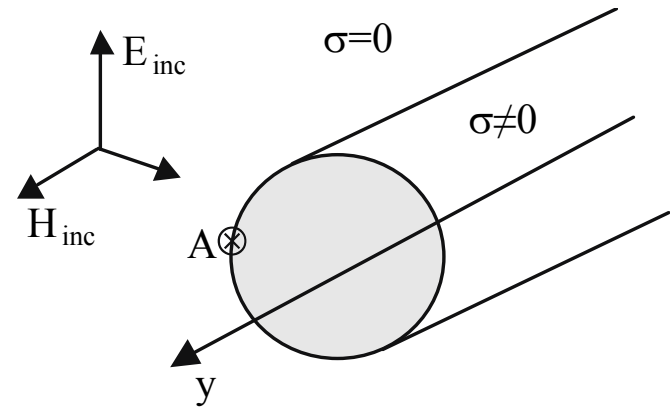

Figure 4. Plane wave impinging on a conducting cylinder. The total magnetic field is computed at point $\mathrm{A}$.

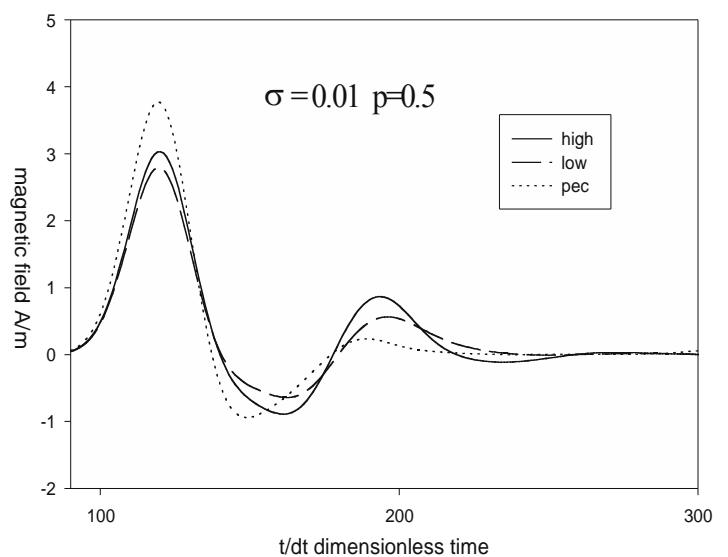

Figure 5. The total magnetic field $[\mathrm{A} / \mathrm{m}]$ at point $\mathrm{A}$ in Figure 4. Comparison of results for PEC, low order SIBC (Leontovich) and high order SIBC (Rytov).

C. Eddy current testing of materials - the question of model reduction.

The previous two examples dealt with geometries that benefited from the use of SIBCs in that the model is reduced and solution is obtained with reduced computational effort. Either one could have been solved numerically without the use of SIBCs albeit with increased computational resources.

There are however applications in which the SIBC is critical to the very ability to solve the problem satisfactorily. An example of this type is afforded by the problem of nondestructive testing in nuclear power plant steam generators. The problem posed on design of tests and on their simulation is the large-scale differences between the various components both dimensionally and more importantly electrically. A basic configuration of a test is shown in Fig. 6a. The requirement is to move the probe, consisting typically of two coils connected differentially to detect small flaws in the stainless steel tube. The support 
plate, made of carbon steel is a feature required for the mechanical support of the tubes. The problems in computation arises from a number of sources:

- The flaws can be as small as a few micrometers with a very small volume or even tight cracks with negligible volume.

- The frequency of tests can be high $-100 \mathrm{kHz}$ to $600 \mathrm{kHz}$ are typical.

- The skin depth in the stainless steel tube is of the order of $0.5 \mathrm{~mm}$ to $0.1 \mathrm{~mm}$

- The skin depth in the support plate is of the order of $50 \mu \mathrm{m}$ to $10 \mu \mathrm{m}$.

- The fields of the probe are weak but can penetrate the stainless steel tube to depths of $10-50 \mathrm{~mm}$.

- The stainless steel tube is $19 \mathrm{~mm}$ in diameter, the coils have an outer diameter of $17 \mathrm{~mm}$ thickness $1 \mathrm{~mm}$, width $1.5 \mathrm{~mm}$ and separation of $2 \mathrm{~mm}$. The gap between the support plate and tube is typically $0.5 \mathrm{~mm}$.

- The probe moves in order to generate a signal. This requires calculation of many probe positions as well as accounting for the velocity of the probe.

Modeling a geometry of this type requires a cylindrical section of about $100 \mathrm{~mm}$ in length and approximately the same diameter to ensure minimum effect of the artificial boundaries on the solution. The combination of small flaws, small skin depths and the need for calculation of minute variations in fields imposes severe restrictions on what can be done practically. Typically a 3D model of this type will require a few million degrees of freedom to obtain a reasonable solution with a large percentage of these in the support plate (up to 10 finite elements per skin depth are required). Elimination of the support plate reduces to model to a manageable size (Fig. 6b) and hence to reasonable solution times [27]. Although the geometrical comparison between Fig. 6a and Fig. $6 \mathrm{~b}$ is not dramatic, computationally it is because the support plate contains in excess of $50 \%$ of the elements hence the geometry in Fig. $6 a$ is much more economical computationally.

To understand the steps involved we will treat here a much simpler problem and its formulation using SIBCs based on the so-called A- $\phi$ formulation. One starts with the following two relations in terms of $\mathbf{A}$ (the magnetic vector potential) and $\phi$ (the electric scalar potential) [28, 29]:

$$
\begin{gathered}
\nabla \times\left(\frac{1}{\mu} \nabla \times \mathbf{A}\right)+\sigma(j \omega \mathbf{A}+\nabla \phi)=\mathbf{J}_{s} \\
\nabla \cdot \sigma(j \omega \mathbf{A}+\nabla \phi)=0
\end{gathered}
$$

It is assumed that any applied current density $\mathbf{J}$ is limited to conductors, that frequency is low and that Coulomb's gauge $(\nabla \cdot \mathbf{A}=0)$ has been applied.

Assuming the geometry has been divided into a mesh with edge elements (with properly defined edges, nodes and facets), we approximate the physical quantities as follows:
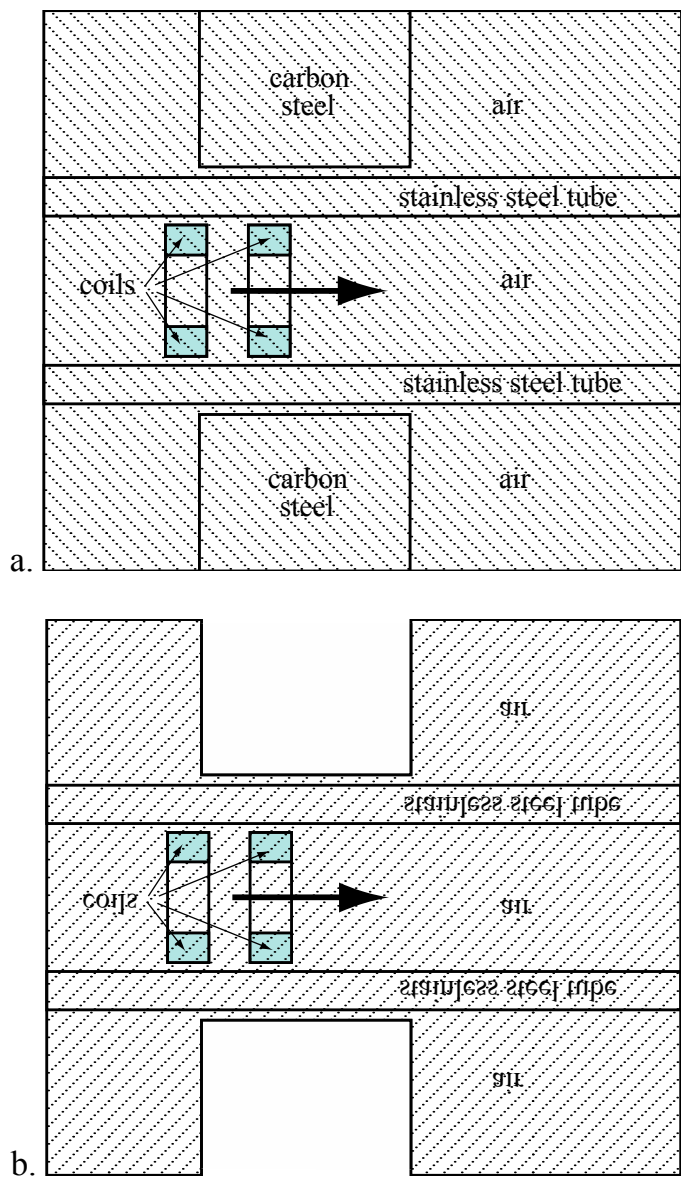

Figure 6. Geometry for an nondestructive evaluation problem. a. Without SIBCs b. With SIBCs. The mesh is indicated by the cross-hatched overlay. The mesh density is defined by the skin depth.

$$
\mathbf{A}=\sum_{e=1}^{N_{e}} A_{e} \mathbf{w}_{e} \quad \phi=\sum_{n=1}^{N_{n}} \phi_{n} w_{n} \quad \mathbf{J}_{s}=\sum_{f=1}^{N_{f}} J_{f} \mathbf{w}_{f}
$$

where $e$ stands for the edges of the mesh, $n$ for its nodes and $f$ for its facets. $\mathbf{w}_{e}$ are interpolation functions on edges, $w_{n}$ are interpolation functions on nodes and $\mathbf{w}_{f}$ are interpolation functions on facets. $N_{e}$ is the number of edges (in an element), $N_{n}$ the number of nodes and $N_{f}$ the number of facets. With these, in conjunction with the Galerkin method after integration by parts we get:

$$
\begin{aligned}
& \int_{v}\left[\left(\frac{1}{\mu} \nabla \times \mathbf{w}_{e}\right) \cdot\left(\nabla \times \mathbf{w}_{e}\right)^{T}\left(A_{e}\right)^{T}+\sigma \mathbf{w}_{e} \cdot j \omega\left(\mathbf{w}_{e}\right)^{T}\left(A_{e}\right)^{T}+\right. \\
& \left.+\sigma \mathbf{w}_{e} \cdot \nabla w_{n} \phi_{n} d v\right] d v+\int_{\Gamma} \mathbf{w}_{e} \cdot[\mathbf{H} \times \hat{\mathbf{n}}] d \Gamma=\int_{v} \mathbf{w}_{e} \sum_{f=1}^{N_{f}} \mathbf{w}_{f} J_{f} d v \\
& \int_{v}\left[\sigma \nabla \mathbf{w}_{n} \cdot j \omega\left(\mathbf{w}_{e}\right)^{T}\left(A_{e}\right)^{T}+\sigma \mathbf{w}_{e} \bullet \nabla w_{n} \phi_{n} d v\right] d v- \\
& \int_{\Gamma}\left(w_{n} \sigma j \omega \mathbf{w}_{e}\left(A_{e}\right)^{T}+w_{n} \sigma \nabla w_{n} \phi_{n}\right) \cdot \hat{\mathbf{n}} d \Gamma=0
\end{aligned}
$$

The left-hand side of (25) and (26) is:

$$
\left[\begin{array}{cc}
\int_{v}\left[\nabla \times \mathbf{w}_{e}\right]^{T} \cdot\left[\nabla \times \mathbf{w}_{e}\right] d v+j \omega \int_{v} \sigma\left[\mathbf{w}_{e}\right]^{T} \cdot\left[\mathbf{w}_{e}\right] d v & \int_{v} \sigma\left[\mathbf{w}_{e}\right]^{T} \cdot\left[\nabla w_{n}\right] d v \\
\int_{v} \sigma\left[\nabla w_{n}\right]^{T} \cdot\left[\mathbf{w}_{e}\right] d v & \frac{1}{j \omega} \int_{v} \sigma\left[\nabla w_{n}\right]^{T} \cdot\left[\nabla w_{n}\right] d v
\end{array}\right]\left[\begin{array}{c}
\mathbf{A} \\
\phi
\end{array}\right]
$$


To introduce the surface impedance, we start with (25) and write for the surface integral:

$$
\int_{\Gamma} \mathbf{w}_{e} \cdot[\mathbf{H} \times \hat{\mathbf{n}}] d \Gamma=\int_{\Gamma} \frac{1}{\mu} \mathbf{w}_{e} \cdot[(\nabla \times \mathbf{A}) \times \hat{\mathbf{n}}] d \Gamma
$$

On the surface itself we write the surface current density as follows:

$$
\mathbf{J}_{s}=\mathbf{H} \times \hat{\mathbf{n}}=\frac{1}{Z_{s}}[\hat{\mathbf{n}} \times \mathbf{E}] \times \hat{\mathbf{n}}=\frac{1}{Z_{s}}[\hat{\mathbf{n}} \times(j \omega \mathbf{A}+\nabla \phi)] \times \hat{\mathbf{n}}
$$

where $Z_{s}$ is the surface impedance. The divergence of this current density must be zero:

$$
\nabla \cdot \frac{1}{Z_{s}}[\hat{\mathbf{n}} \times(j \omega \mathbf{A}+\nabla \phi)] \times \hat{\mathbf{n}}=0
$$

Writing this in the Glerkin form we have on the impedance surface:

$$
\int_{\Gamma} \frac{1}{Z_{s}}\left[\hat{\mathbf{n}} \times\left(\nabla w_{n} \cdot j \omega\left(\mathbf{w}_{e}\right)^{T}\left(A_{a}\right)^{T}+\nabla w_{n} \cdot \nabla w_{n} \phi\right)\right] \times \hat{\mathbf{n}} d \Gamma=0
$$

The term $\mathbf{H} \times \hat{\mathbf{n}}$ in (25) is replaced with the right hand side of (29) and we have for the surface term:

$$
\int_{\Gamma} \mathbf{w}_{e} \cdot[\mathbf{H} \times \hat{\mathbf{n}}] d \Gamma=\int_{\Gamma} \frac{1}{Z_{s}} \mathbf{w}_{e} \cdot\left[j \omega\left(\mathbf{w}_{e}\right)^{T}\left(A_{e}\right)^{T}+\nabla w_{n} \phi\right] d \Gamma
$$

Therefore, on the impedance surface the elemental matrix contribution is:

$$
\left[\begin{array}{cc}
\int_{\Gamma} \frac{1}{Z_{s}} \mathbf{w}_{e} \cdot j \omega\left(\mathbf{w}_{e}\right)^{T} d \Gamma & \int_{\Gamma} \frac{1}{Z_{s}} \mathbf{w}_{e} \cdot \nabla w_{n} d \Gamma \\
\int_{\Gamma} \frac{1}{Z_{s}} \nabla w_{n} \cdot j \omega\left(\mathbf{w}_{e}\right)^{T} d \Gamma & \int_{\Gamma} \frac{1}{Z_{s}} \nabla \mathbf{w}_{n} \cdot \nabla w_{n} d \Gamma
\end{array}\right]\left[\begin{array}{c}
\mathbf{A} \\
\phi
\end{array}\right]
$$

Note that the surface term in (26) does not contribute to the right hand side since the normal component of the current density on the surface is zero.

The contribution of (33) is evaluated on all elements on the impedance surface and placed into the right hand side of the matrix system before solving.

The $2^{\text {nd }}$ order surface impedance boundary condition (from (2)) may be written as:

$$
Z_{s}=j \omega \mu\left[(j \omega \sigma \mu)^{-1 / 2}-(j \omega \sigma \mu)^{-1} \frac{d_{k}-d_{3-k}}{2 d_{k} d_{3-k}}\right], k=1,2
$$

The $1^{\text {st }}$ order surface impedance is obtained by removing the $2^{\text {nd }}$ term:

$$
Z_{s}=j \omega \mu(j \omega \sigma \mu)^{-1 / 2}=\sqrt{\frac{j \omega \mu}{\sigma}}=\frac{1+j}{\sigma \delta}
$$

Although a $3^{\text {rd }}$ order SIBC may also be used, it involves second order derivatives (see (1)) and since the finite elements most often used are first order, the second derivatives are zero. In other words, $3^{\text {rd }}$ order SIBCs require at least $2^{\text {nd }}$ order finite elements.

As a simple test case, the magnetic field of a small coil over a conducting sheet is calculated. This is a classical problem in nondestructive testing in which either the field or the ac impedance of the coil is monitored as an indication of flaws or variations in material properties. The conducting sheet is $3 \mathrm{~mm}$ thick but very large in the other dimensions. Its conductivity is $10^{7} \mathrm{~S} / \mathrm{m}$ and its relative permeability 70 , representing carbon steel. The coil is small, with a crosssection of $1 \mathrm{~mm}$ by $1 \mathrm{~mm}$, with inner radius of $3 \mathrm{~mm}$ and placed $1 \mathrm{~mm}$ above the sheet (Fig. 7). The coil is made of 50 turns carrying a unit current at $100 \mathrm{kHz}$.

air \begin{tabular}{c|c|c|}
$4 \mathrm{~mm}$ \\
$1 \mathrm{~mm}$
\end{tabular}

Figure 7. Geometry of a simple testing configuration.

This test problem does not actually require the use of SIBCs and because the surfaces are flat, only 1st order SIBCs are needed (no curvature). The simplicity of the geometry allows comparison with FEM solution in the absence of SIBCs as well as to explore the errors involved. The solution without SIBCs is shown in Fig. 8a. Figure 8b shows the solution with the first order SIBC applied on the interface between the conductor and air.
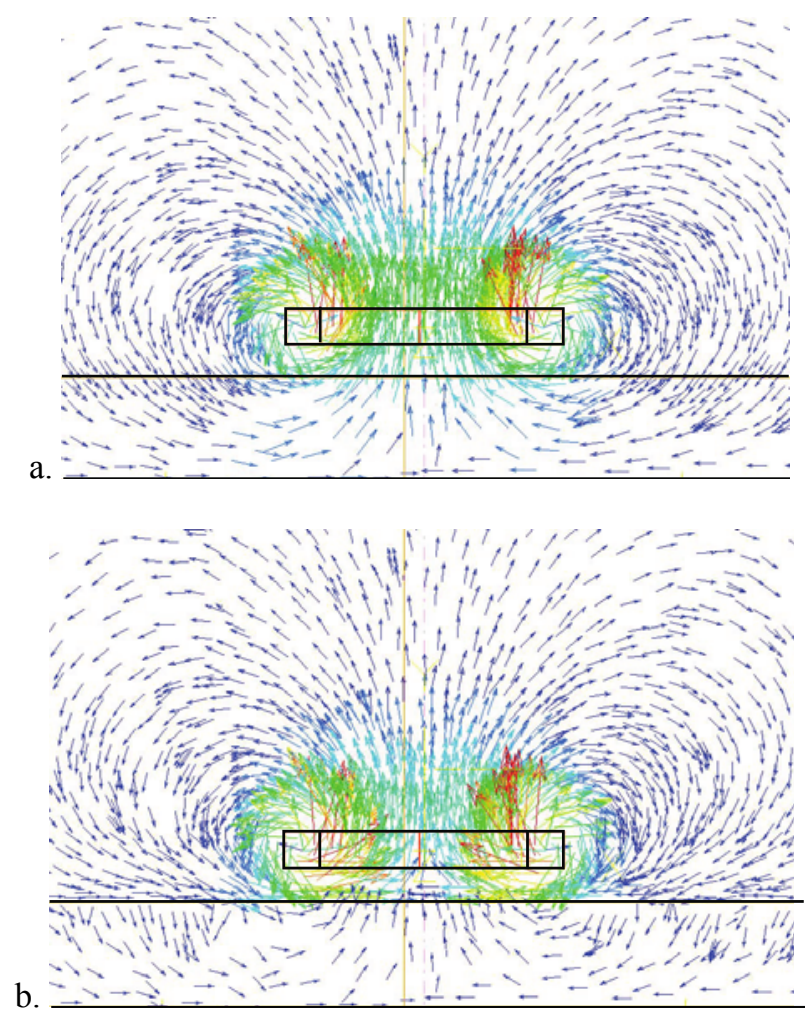

Figure 8. a. FEM solution for the geometry in Fig. 1 without SIBC. b. With SIBC imposed on the interface between conductor and air. 
The flux with and without SIBCs are compared, with the surface impedance condition imposed on the upper surface of the conductor. Normally, the conductor would be excluded from the mesh entirely - that is one of the reasons for introduction of surface impedance boundary conditions but in this case it was deemed useful to leave the mesh unchanged to show that the surface impedance boundary condition does not eliminate the fields in the conductor entirely - it only reduces them to insignificant levels. Although the two figures look similar, there are subtle changes in the field, particularly in the gap between the coil and the conductor. In particular, since the SIBC is internal to the geometry modeled, one can see numerical noise in the volume of the conductor. The fields are very low but, nevertheless, are not null.

A better indication of the changes in the fields, are the numerical values of flux shown in Table II, for various conductivities. As expected, the error increases with the decrease in conductivity since as the frequency decreases the skin depth increases and hence the ratio between skin depth and the characteristic dimension of the geometry (the thickness of the conductor) decreases.

TABLE II. ERROR IN CALCULATION OF FLUX IN THE COILS AS A FUNCTION OF CONDUCTIVITY OF THE PLATE.

\begin{tabular}{|c|c|c|c|}
\hline Conductivity & $\begin{array}{c}\text { Flux without } \\
\text { SIBC }\end{array}$ & $\begin{array}{c}\text { Flux with } \\
\text { SIBC }\end{array}$ & $\begin{array}{c}\text { \% change } \\
\text { (error) }\end{array}$ \\
\hline $10^{7} \mathrm{~S} / \mathrm{m}$ & 9.33948 & 9.22142 & 1.28 \\
\hline $10^{6} \mathrm{~S} / \mathrm{m}$ & 9.49206 & 9.22142 & 2.93 \\
\hline $10^{5} \mathrm{~S} / \mathrm{m}$ & 9.57937 & 9.22142 & 3.88 \\
\hline $10^{4} \mathrm{~S} / \mathrm{m}$ & 9.58843 & 9.221419 & 3.99 \\
\hline
\end{tabular}

\section{Coupled problems}

Surface impedance boundary conditions apply to a variety of coupled, nonlinear, layered and nonhomogeneous problems. To demonstrate its utility in design and computation we discuss here a coupled electromagneticthermal problem. The equation governing the field distribution in the conductor is supplemented by the heat transfer equation:

$$
c \rho \frac{\partial T}{\partial t}=\nabla \cdot(\lambda \nabla T)+\frac{J^{2}}{\sigma}
$$

where the coupling is through the current density $J . \sigma$ and $\lambda$ are the electric and thermal conductivities, $c$ and $\rho$ are the specific heat and mass density respectively. With $\mathbf{J}=\nabla \times \mathbf{H}$ from Maxwell's equations, the coupling is explicit:

$$
\frac{\partial \tilde{T}}{\partial \tilde{t}}=\tilde{\varepsilon}^{2} \tilde{\nabla} \cdot(\tilde{\lambda} \tilde{\nabla} \tilde{T})+\tilde{\sigma}^{-1}(\tilde{\nabla} \times \tilde{\mathbf{H}})^{2}
$$

For convenience and ease of handling, we re-write the equation in nondimensional variables [3] using scale factors for the variables:

$$
\frac{\partial \tilde{T}}{\partial \tilde{t}}=\tilde{\varepsilon}^{2} \tilde{\nabla} \cdot(\tilde{\lambda} \tilde{\nabla} \tilde{T})+\tilde{\sigma}^{-1}(\tilde{\nabla} \times \tilde{\mathbf{H}})^{2}
$$

where $\tilde{\varepsilon}=\left(\lambda^{*} \sigma^{*} \mu /(c \rho)\right)^{1 / 2}$ and $\lambda^{*}=\lambda\left(\mathrm{T}^{*}\right), \sigma^{*}=\sigma\left(\mathrm{T}^{*}\right)$ and $\mathrm{T}^{*}$ is a characteristic temperature used as a scale factor. The permeability $\mu$ is introduced by the skin depth through the scaling process.

For a first order approximation (Leontovich condition) and recalling that the variations in the magnetic field and in temperature are only with respect to the perpendicular coordinate $\eta$, we get:

$$
\frac{\partial \tilde{T}}{\partial \tilde{t}}=\tilde{\varepsilon}^{2} \frac{\partial}{\partial \tilde{\eta}}\left(\tilde{\lambda} \frac{\partial \tilde{T}}{\partial \tilde{\eta}}\right)+\tilde{\sigma}^{-1} \sum_{i=1}^{2}\left(\frac{\partial \tilde{H}_{\xi_{i}}}{\partial \tilde{\eta}}\right)^{2}
$$

The relations between the tangential components of the electric and magnetic fields are:

$$
\begin{aligned}
& \tilde{p} \frac{\partial \tilde{H}_{\xi_{k}}}{\partial \tilde{\eta}}=(-1)^{3-k} \tilde{\sigma} \tilde{E}_{\xi_{3-k}}, \mathrm{k}=1,2 \\
& \frac{\partial \tilde{E}_{\xi_{3-k}}}{\partial \tilde{\eta}}=(-1)^{3-k} \tilde{p} \frac{\partial \tilde{H}_{\xi_{k}}}{\partial \tilde{t}}, \mathrm{k}=1,2
\end{aligned}
$$

where $\tilde{p}=\delta / D$ and $D$ is the characteristic dimension in the geometry $(D>>\delta)$.

The diffusion of the magnetic field into the conductor can be written from (40) and (41) as:

$$
\frac{\partial}{\partial \tilde{\eta}}\left(\tilde{\sigma}^{-1} \frac{\partial \tilde{H}_{\xi_{k}}}{\partial \tilde{\eta}}\right)=\frac{\partial \tilde{H}_{\xi_{k}}}{\partial \tilde{t}}, \mathrm{k}=1,2
$$

(40) and (41) are solved together with the boundary and initial conditions:

$$
\begin{gathered}
\tilde{\eta}=0: ; \tilde{H}=\tilde{H}^{b}, \frac{\partial \tilde{T}}{\partial \tilde{\eta}}=\gamma\left(\tilde{T}-\frac{T_{\infty}}{T^{*}}\right) \\
\tilde{\eta} \rightarrow \infty: \tilde{H} \rightarrow 0, \tilde{T} \rightarrow T_{\infty} / T^{*} \\
\tilde{t}=0: \tilde{H}=0, \tilde{T}=T_{\infty} / T^{*}
\end{gathered}
$$

where $T_{\infty}$ is the reference temperature. Returning to dimensional variables results in the following:

$$
\begin{gathered}
\frac{\partial}{\partial \eta}\left((\sigma \mu)^{-1} \frac{\partial H_{\xi_{k}}}{\partial \eta}\right)=\frac{\partial H_{\xi_{k}}}{\partial t} \quad k=1,2 \\
c \rho \frac{\partial T}{\partial t}=\frac{\partial}{\partial \eta}\left(\lambda \frac{\partial T}{\partial \tilde{\eta}}\right)+\sigma^{-1} \sum_{i=1}^{2}\left(\frac{\partial H_{\xi_{i}}}{\partial \eta}\right)^{2} \quad k=1,2 \\
E_{\xi_{3-k}}^{b}=(-1)^{k} \mu \frac{\partial}{\partial t} \int_{0}^{\infty} H_{\xi_{k}} d \eta
\end{gathered}
$$


As an example, consider the configuration in Fig. 8 in which the surface current on the conductors is required. The current pulse applied to the conductor is also shown. The following temperature dependencies of conductivity and thermal conductivity are assumed:

$$
\sigma=\frac{\sigma_{\infty}}{1+\alpha T} \quad \lambda=\chi \sigma T \lambda=\chi \sigma T
$$

where the coefficients $\alpha$ and $\chi$ can be assumed to be constant. Substitution of these relations into (46) and (47) leads to the following SIBC:

$$
\left\{\begin{array}{c}
\frac{\partial}{\partial \tilde{\eta}}\left(\left(1+\alpha T^{*} \tilde{T}\right) \frac{\partial \tilde{H}_{\xi_{k}}}{\partial \tilde{\eta}}\right)=\sigma_{\infty} \mu \frac{\partial \tilde{H}_{\xi_{k}}}{\partial \tilde{t}} \\
c \rho \frac{\partial T}{\partial t}=\chi \sigma_{\infty} \frac{\partial}{\partial \eta}\left(\frac{T}{1+\alpha T} \frac{\partial T}{\partial \eta}\right)+\sigma^{-1} \sum_{i=1}^{2}\left(\frac{\partial H_{\xi_{i}}}{\partial \eta}\right)^{2}
\end{array}\right.
$$
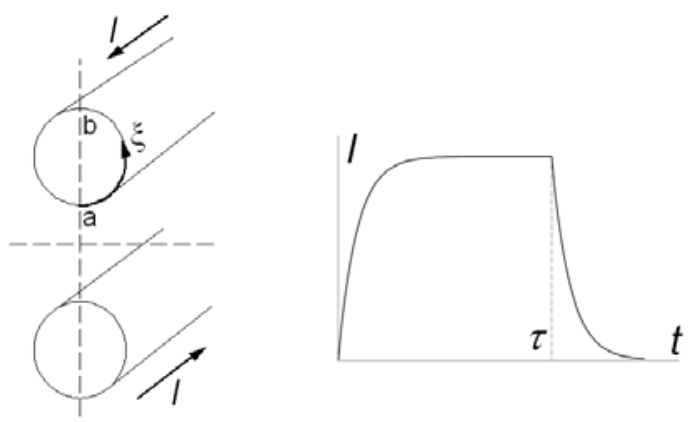

Figure 9. Geometry for a coupled electromagnetic-thermal problem. The inset shows the current pulse applied.

Figure 10 shows distributions of the surface current density $K$, obtained using different approximations of the electromagnetic field diffusion into the conductors, as a function of the surface coordinate $\xi$. The origin is at point $a$ and proceeds around the contour, ending at point $b$ (due to symmetry only half the contour around the cross section of one conductor is shown).

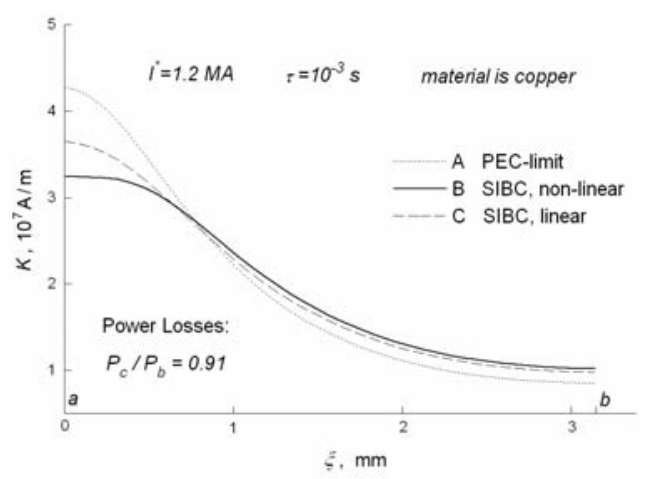

Figure 10. Distribution of the surface current density along the half contour around one of the conductors in Fig. 9.

Curve $A$ was obtained using the perfect electrical conductor (PEC) limit (the electromagnetic field diffusion is neglected). Curve $C$ was obtained using the classical Leontovich SIBC in which the conductors' properties are assumed to be constant and curve $B$ was obtained using the non-linear SIBC in (50). As in the previous examples, the advantage of the use of SIBCs is clear but in this case the difference between the use of the linear and nonlinear SIBCs is also emphasized.

\section{CONCLUSIONS}

The use of surface impedance boundary conditions of various order to the problem of computation and computer aided design has been introduced and a few examples to its use and advantages pointed out. Although the development of appropriate SIBCs and their formulations can be complex, their use is simple. The result is an economical approach to numerical computation and together with error control, the methods shown here can result in accurate solutions. Surface impedance boundary conditions can be used at low frequencies as long as the ratio between the skin depth and the characteristic dimension of the structure is small. The size of this ratio governs the error in the calculation. The examples presented point to a wide range of practical applications at low frequencies.

\section{REFERENCES}

[1] S.A. Schelkunoff, "The impedance concept and its application to problems of reflection, radiation, shielding and power absorption," Bell Systems Technical Journal, Vol. 17, pp. 17-48, 1938.

[2] M. A. Leontovich, "On the approximate boundary conditions for the electromagnetic field on the surface of well conducting bodies," in Investigations of Radio Waves, B.A. Vvedensky Ed., Moscow: Acad. of Sciences of USSR (in Russian), pp. 5-12, 1948.

[3] S. Yuferev and N. Ida, Surface Impedance Boundary Conditions: A Comprehensive Approach. Boca Raton: CRC Press, 2010.

[4] P. Dular, C. Geuzaine and J. Gyselinck, "Surface impedance boundary conditions in dual time domain finite element formulations, "IEEE Transactions on Magnetics, Vol. 46 No. 8, pp. 3524-3531, 2010

[5] S. Cruciani, M. Feliziani and M. Okoniewski, "Efficient low order approximation for surface impedance boundary conditions in finitedifference time domain method,“ IEEE Transactions on Magnetics, Vol. 48, No. 2, pp. 271-274, 2012

[6] R. Sabariego, C. Geuzaine, P. Dular and J. Gyselinck, "Time-domain surface impedance boundary conditions enhanced by coarse volume finite element discretization," IEEE Transactions on Magnetics, Vol. 48, No. 2, pp. 631-634, 2012

[7] A. D. U. Jafri, Q. A. Naqvi and K. Hongo, "Scattering of electromagnetic plane wave by a circular disk with surface impedance," Progress In Electromagnetics Research, Vol. 127, pp. 501-522, 2012

[8] M. Kunze, "Surface impedances for planar conductors in volume discretization methods without frequency limitations," Microwave Conference (GeMIC), pp. 1 - 4, 2011

[9] I.V. Lindell and A. Sihvola, "Mixed-Impedance Boundary Conditions," IEEE Transactions on Antennas and Propagation, Vol. 59, No. 1, pp. 1580-1586, 2011

[10] S. Yuferev and N. Ida, "Selection of the Surface Impedance Boundary Conditions for a Given Problem," IEEE Transactions on Magnetics, Vol. 35, No. 3, pp. 1486-1489, May 1999.

[11] S. Yuferev and N. Ida, "Time domain surface impedance concept for low frequency electromagnetic problems - Part I: derivation of high order surface impedance boundary conditions in the time domain," IEE Proceedings - Science, Measurement and Technology, Vol. 152, No. 4, pp. 175-185, July 2005.

[12] S. Barmada, L. Di Rienzo, N. Ida, and S. Yuferev, "The time domain surface impedance concept for low frequency electromagnetic problems - Part II: application to transient skin and proximity effect problems in cylindrical conductors," IEE Proceedings - Science, Measurement and Technology, Vol. 152, No. 5, pp. 207-216, Sept. 2005.

[13] S. Schmid, N. Chavannes, N, Kuster, E. Okoniewska, D. Pasalic and M. Okoniewski, "Advanced surface impedance boundary conditions in EM-FDTD," Proceedings of the 2011 IEEE International Symposium on Antennas and Propagation, pp. 2319-2311, 2011 
[14] J. J. Akerson, M. A. Tassoudji, Y. E. Yang, J. A. Kong, "Finite difference time domain (FDTD) impedance boundary condition for thin finite conducting sheets," Progress In Electromagnetics Research, PIER 31, pp. 1-30, 2001

[15] S. M. Rytov, "Calcul du skin-effet par la méthode des perturbations," Journal of Physics, 2 (3), 1940, 233-242.

[16] L. Di Rienzo, S. Yuferev, and N. Ida, "Computation of the impedance matrix of multiconductor transmission lines using high order surface impedance boundary conditions," IEEE Trans. Electromagn. Compat., Vol. 50, No. 4, November 2008, pp. 974-984.

[17] S. Yuferev and L. Di Rienzo, "Surface impedance boundary conditions in terms of various formalisms," IEEE Trans. Magn., Vol. 46, No. 9, September 2010, pp. 3617-3628.

[18] S. Yuferev, L. Di Rienzo and N. Ida, "Surface Impedance Boundary Conditions for Finite Integration Technique," in IEEE Transactions on Magnetics, Vol. 42, No. 4, April 2006, pp. 823-826.

[19] S. Yuferev, N. Farahat and N. Ida, "Use of the Perturbation Technique for Implementation of Surface Impedance Boundary Conditions for the FDTD Method," IEEE Transactions on Magnetics, Vol. 36, No. 4, July 2000, pp. 942-945.

[20] S. Yuferev and N. Ida, "Efficient Implementation of the TimeDomain Surface Integral Boundary Condition for the Boundary Integral Method," IEEE Transactions on Magnetics, Vol. 34 No. 5, pp. 2763-2666, Sept. 1998.

[21] S. Yuferev and N. Ida, "Impedance Boundary Conditions of High Order Approximation for Electromagnetic Transient Scattering Problems," Proceedings of the 10th International Conference on Antennas and Propagation, Edinburgh, April 12-17, 1997), IEE Publication No. 426, pp.1,306-1,309, 1997.
[22] M. Cao and P. P. Biringer, "BIE formulation for skin and proximity effect problems of parallel conductors," IEEE Trans. Magn., Vol. 26 No. 5, pp. 2768-2770, Sept. 1990.

[23] C. A. Brebbia, The Boundary Element Method for Engineers. London: Pentech Press, 1980.

[24] S. Yuferev and N. Ida, "Selection of the Surface Impedance Boundary Conditions for a Given Problem," IEEE Transactions on Magnetics, Vol. 35, No. 3, pp. 1486-1489, May 1999.

[25] N. Farahat, S. Yuferev and N. Ida, "High order surface impedance boundary conditions for the FDTD method," IEEE Transactions on Magnetics, Vol. 37, No. 5, September 2001, pp. 3242-3245.

[26] S. Yuferev L. Proekt and N. Ida, "Surface impedance boundary conditions near corners and edges: rigorous consideration," in IEEE Transactions on Magnetics, Vol. 37 No. 5, Sep 2001, pp. 3465-3468.

[27] O. Moreau, V. Costan, J.-M. Devinck, and N. Ida, "Finite Element Modeling of Support Plate Clogging-up in Nuclear Plant Steam Generator," Proceedings of the 8th International Conference on NDE in Relation to Structural Integrity for Nuclear and Pressurised Components, 29 September to 1 October 2010, Berlin, Germany (PDF only).

[28] Y. Le Menach, "Contribution a la modelisation numerique tridimensionnelle des systemes electrotechniques," $\mathrm{Ph}$.D. dissertation, Universite de Lille 1, 1999.

[29] N. Ida, Y. Lemenach, and T. Henneron, "High Order Surface Impedance Boundary Conditions with the A- $\Phi$ Formulation," Facta Universitatis: Electronics and Energetics, vol. 24, No. 2, August 2011 pp. 147-155. 